\title{
BESSEL FUNCTIONS FOR AXISYMMETRIC ELASTICITY PROBLEMS OF THE ELASTIC HALF SPACE SOIL: A POTENTIAL FUNCTION METHOD
}

\author{
C. C. Ike ${ }^{1,}{ }^{*}$, H. N. Onah ${ }^{2}$ and C. U. Nwoji ${ }^{3}$

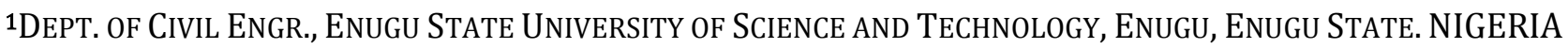 \\ 2,3DEPARTMENT OF Civil ENGINEERING, UNiverSity OF NigERIA, NSUKKA. ENUGU STATE. NIGERIA \\ Email addresses:1 charles.ike@esut.edu.ng, 2hyginus.onah@unn.edu.ng, ${ }^{3}$ clifford.nwoji@unn.edu.ng
}

\begin{abstract}
Elasticity problems are formulated using displacement methods or stress methods. In this paper a displacement formulation of axisymmetric elasticity problem is presented. The formulation uses the Boussinesq-Papkovich - Neuber potential function. The problem is then solved by assuming Boussinesq - Papkovich - Neuber potential functions in the form of Bessel functions of order zero and of the first kind. The potential functions are then made to satisfy the governing field equations and the associated boundary conditions for the particular problem of a point load at the origin of the semiinfinite linear elastic isotropic soil mass. The unknown parameters of the function are thus determined and used to find the stresses, strains and displacement fields in the loaded soil. The results obtained were identical with the results obtained by Boussinesq.
\end{abstract}

Keywords: Axisymmetric elasticity problem, Boussinesq - Papkovich - Neuber potential function, Bessel functions of order zero and of the first kind, semi-infinite soil mass, displacement formulation, stress formulation.

\section{INTRODUCTION}

Axisymmetric elasticity problems of the half-space are elasticity problems where the loading is rotationally symmetric about an axis perpendicular to the surface. The cylindrical coordinate system is the natural coordinate system of choice for the description and analysis of axisymmetric problems. The problems are defined in terms of the radial, $r$ circumferential, $\theta$ and depth, $z$ coordinate variables; and the corresponding displacements defined as $u_{r}, u_{\theta}$, and $u_{z}$ (or, $u, v$, and $w$ respectively) where $u_{r}, u_{\theta}$, and $u_{z}$ are displacement components in the radial, circumferential and depth coordinate directions, respectively. The horizontal surface of the half-space is usually defined to lie on the $r \theta$ plane and the vertical, $z$ axis is directed into the halfspace. For axisymmetric problems, the stresses and displacements are independent of the circumferential coordinate, $\theta$ and when there are no applied torques, the circumferential displacement components vanish. In the present study, the half-space is considered homogeneous, isotropic and linear elastic and the load is assumed to be applied statically. The deformations are assumed to be small; hence small deformation assumptions are applied. The study of such an idealized elastic continuum is the subject matter of the classical theory of elasticity and in the case of vertical applied loads, was first studied by Boussinesq. This study is significant because from point load solutions, stresses and displacements due to any kind of distributed load applied to the surface of a soil mass considered homogeneous, isotropic, linear elastic half-space could be obtained by integration over the loaded region (area) with the point load solution considered as the Green function [1].

\subsection{Bessel Functions and Axisymmetric Problems}

The Bessel's equations are commonly encountered in partial differential equations in bodies having cylindrical symmetry. Bessel functions are also encountered in the solution of Laplace equations in cylindrical coordinates [2]. In advanced mathematics, Bessel functions denoted by $J_{n}(x)$ are canonical solutions of Bessel's differential equation:

$$
x y^{\prime \prime}+x y^{\prime}+\left(x^{2}-n^{2}\right) y=0
$$

where, $y(x)$ and $y^{\prime}=\frac{d y}{d x}, y^{\prime \prime}=\frac{d^{2} y}{d x^{2}}$ and $n$ is called the order of the Bessel function; and can be a real or complex number. The most common case is for integer values of $n$. Solutions of the Laplace equation in cylindrical coordinates are Bessel functions of integer order, frequently called cylinder functions. A more general parametric representation of the Bessel equation is 
obtained by replacing $x$ with $\lambda x$ where $\lambda$ is a parameter, to obtain [2]:

$$
x^{2} y^{\prime \prime}+x y^{\prime}+\left(\lambda^{2} x^{2}-n^{2}\right) y=0
$$

\subsection{Research Aim and Objectives}

The research aim and objectives include:

(i) To present the general governing partial differential equations for the axisymmetric elasticity problem of linearly elastic, homogenous, isotropic materials.

(ii) To present a displacement potential function formulation using the Boussinesq - Papkovich Neuber potential function for the axisymmetric problem

(iii) To solve the axisymmetric elasticity problem of homogenous soil of semi-infinite extent loaded by a point load, $\mathrm{P}$ applied at the origin using Boussinesq Papkovich - Neuber potential functions assumed in the form of Bessel functions.

\section{LITERATURE REVIEW}

Two formulations of elasticity problem are found in literature; namely displacement and stress formulations[3], [4]. In displacement formulation, displacements are the primary unknown variables, from which strain and stress fieldsare found by using the strain-displacement relations and the material constitutive laws. In stress formulation, stresses are the primary unknown variables and from the stress components, strain components are found from the stress-strain laws and then displacement components found from the kinematic relations. In stress formulationone will always make use of the compatibility conditions in the solutions process, in order to determine compatible displacement fields.

\subsection{Displacement Formulation of Three Dimensional(3D) Elasticity Problems}

Navier obtained the governing equations of three dimensional elasticity problems in terms of displacements by combining stress equations of equilibrium with the stress displacement equations as follows $[5,6,7]$ :

$$
\begin{aligned}
& \nabla^{2} u+\left(\frac{1}{1-2 \mu}\right) \frac{\partial}{\partial x}\left(\frac{\partial u}{\partial x}+\frac{\partial v}{\partial y}+\frac{\partial w}{\partial z}\right)+\frac{F_{x}}{G}=0 \\
& \nabla^{2} v+\left(\frac{1}{1-2 \mu}\right) \frac{\partial}{\partial y}\left(\frac{\partial u}{\partial x}+\frac{\partial v}{\partial y}+\frac{\partial w}{\partial z}\right)+\frac{F_{y}}{G}=0 \\
& \nabla^{2} w+\left(\frac{1}{1-2 \mu}\right) \frac{\partial}{\partial z}\left(\frac{\partial u}{\partial x}+\frac{\partial v}{\partial y}+\frac{\partial w}{\partial z}\right)+\frac{F_{z}}{G}=0 \\
& \text { where, } \quad \nabla^{2}=\frac{\partial^{2}}{\partial x^{2}}+\frac{\partial^{2}}{\partial y^{2}}+\frac{\partial^{2}}{\partial z^{2}}
\end{aligned}
$$

$\nabla^{2}$ is the Laplacian operator, $u, \quad v, \quad w$ are the displacement components in the $x, y$, and $z$ coordinate directions, respectively, $\mu$ is the Poisson's ratio, $G$ is the shear modulus, and $F_{x}, F_{y}, F_{z}$ are the $x, y$, and $z$ components of the body force.

Solutions of the Navier-Lame differential equations satisfying the boundary conditions would yield the displacement components, $u, v$, and $w$. Navier displacement formulation is a system of three coupledpartial differential equations in terms of three unknowndisplacement components; $u, v$ and $w$. In the displacement formulation, the compatibility of deformations is guaranteed automatically since the displacements are determined such that boundary conditions and equilibrium conditions are identically satisfied.

\subsection{Stress Formulation}

The Beltrami-Mitchell equations for stress formulation ofthreedimensional elasticity problems are a system of six partial differential equations [4], [5]

$$
\begin{gathered}
\nabla^{2} \sigma_{x x}+\frac{1}{1+\mu} \frac{\partial^{2} I_{1}}{\partial x^{2}}=\frac{-\mu}{1-\mu}\left(\frac{\partial F_{x}}{\partial x}+\frac{\partial F_{y}}{\partial y}+\frac{\partial F_{z}}{\partial z}\right)-2 \frac{\partial F_{x}}{\partial x} \\
=0 \\
\nabla^{2} \sigma_{y y}+\frac{1}{1+\mu} \frac{\partial^{2} I_{1}}{\partial y^{2}}=\frac{-\mu}{1-\mu}\left(\frac{\partial F_{x}}{\partial x}+\frac{\partial F_{y}}{\partial y}+\frac{\partial F_{z}}{\partial z}\right)-2 \frac{\partial F_{y}}{\partial y} \\
=0 \\
\nabla^{2} \sigma_{z z}+\frac{1}{1+\mu} \frac{\partial^{2} I_{1}}{\partial z^{2}}=\frac{-\mu}{1-\mu}\left(\frac{\partial F_{x}}{\partial x}+\frac{\partial F_{y}}{\partial y}+\frac{\partial F_{z}}{\partial z}\right)-2 \frac{\partial F_{z}}{\partial z} \\
=0
\end{gathered}
$$

where $\mathrm{I}_{1}$ is the stress invariant

$$
\begin{aligned}
\nabla^{2} \sigma_{x y}+\frac{1}{1+\mu} \frac{\partial^{2} I_{1}}{\partial x \partial y} & =-\left(\frac{\partial F_{x}}{\partial y}+\frac{\partial F_{y}}{\partial x}\right) \\
\nabla^{2} \sigma_{y z}+\frac{1}{1+\mu} \frac{\partial^{2} I_{1}}{\partial y \partial z} & =-\left(\frac{\partial F_{y}}{\partial z}+\frac{\partial F_{z}}{\partial y}\right) \\
\nabla^{2} \sigma_{z x}+\frac{1}{1+\mu} \frac{\partial^{2} I_{1}}{\partial z \partial x} & =-\left(\frac{\partial F_{x}}{\partial z}+\frac{\partial F_{z}}{\partial x}\right)
\end{aligned}
$$

Where

$$
I_{1}=\sigma_{x x}+\sigma_{y y}+\sigma_{z z}
$$

$\sigma_{x x}, \sigma_{y y}, \sigma_{z z}$ are the normal stresses, $\sigma_{x y}, \sigma_{y z}$, and $\sigma_{z x}$ are the shear stresses, $F_{x}, F_{y}$ and $F_{z}$ are the body force components in the $X, y$, and $z$ directions per unit volume. Solutions of the Beltrami - Mitchell stress equations for given boundary conditions would yield six stress components $\sigma_{x x}, \sigma_{y y}, \sigma_{z z}, \sigma_{x y}, \sigma_{y z}$ and $\sigma_{z x}$. Analytical rigorous solutions for three dimensional elasticity problems are very difficult to obtain using mathematically exact methods and the number of three dimensional elasticity problems that have thus far been solved is very small.

\subsection{Airy Stress Potential Functions $\phi(r)$}

Airy's stress potential functions $\phi(r)$ are scalar fields defined in the structures' domain that are solutions of 
the biharmonic equation, that is the compatibility equation, and that gives stresses that satisfy the differential equations of equilibrium and hence provide compatible strains through Hooke's stress - strain law.

For any Airy stress function, $\phi(r)$ the Cartesian coordinate stresses $\left(\sigma_{x x}, \sigma_{y y}\right.$, and $\left.\tau_{x y}\right)$ are defined by the following:[8]

$$
\begin{gathered}
\sigma_{x x}=\frac{\partial^{2} \phi}{\partial y^{2}} \\
\sigma_{y y}=\frac{\partial^{2} \phi}{\partial x^{2}} \\
\tau_{x y}=\frac{-\partial^{2} \phi}{\partial x \partial y}
\end{gathered}
$$

or

$$
\begin{gathered}
\sigma_{x x}=\frac{\partial^{2} \phi}{\partial y^{2}}+V \\
\sigma_{y y}=\frac{\partial^{2} \phi}{\partial x^{2}}+V \\
\tau_{x y}=\frac{-\partial^{2} \phi}{\partial x \partial y} \\
F_{x}=-\frac{\partial V}{\partial x} \\
F_{y}=-\frac{\partial V}{\partial y}
\end{gathered}
$$

where $V(x, y)$ is the body force potential

$\mathrm{F}_{\mathrm{x}}$ and $\mathrm{F}_{\mathrm{y}}$ are body force components in the $x$ and $y$ coordinate directions

$$
F=-\nabla \mathrm{V}
$$

In plane strain, stress based governing equation is:

$$
\nabla^{4} \phi=\nabla^{2} \nabla^{2} \phi=-\left(\frac{1-2 \mu}{1-\mu}\right) \nabla^{2} V
$$

In plane stress the stress based governing equation is:

$$
\nabla^{2} \nabla^{2} \phi=\nabla^{4} \phi=-(1-\mu) \nabla^{2} V
$$

A series of simple states of stress may be derived from the stress function expressed in polynomial form as follows:

$$
\begin{aligned}
\phi(\mathrm{x}, \mathrm{y})=a_{0} x^{2}+ & a_{1} x y+a_{2} y^{2}+a_{3} x^{3}+a_{4} x^{2} y \\
& +a_{5} x y^{2}+a_{6} y^{3}+a_{7} x^{3} y+a_{8} x y^{3}(25)
\end{aligned}
$$

where, $a_{0}, a_{1}, a_{2} \ldots a_{8}$ are constants. Each term in this stress function satisfies the compatibility equation:

$$
\nabla^{2} \nabla^{2} \phi(x, y)=0
$$

Where:

$$
\nabla^{2}=\frac{\partial^{2}}{\partial x^{2}}+\frac{\partial^{2}}{\partial y^{2}}
$$

$\nabla^{2}$ is the two dimensional Laplace operator, or the two dimensional Laplacian.

Solutions to plane strain and plane stress problems of elasticity can be determined using Airy's stress function methods. The Airy's stress function method reduces the general formulation to a single partial differential equation in terms of a single unknown, called the Airy's stress function $\phi(X, Y)$, which is a scalar field expressed in terms of the space coordinate variables $x$ and $y$ for problems on the $x, y$ plane. The method is based on the general idea of developing a mathematical/analytical representation of the stress field that will automatically satisfy the differential equations of equilibrium.

\subsection{Airy'sStress Potential Function In Polar Coordinates}

The Airy's stress potential functions $\phi(r, \theta)$ in plane polar coordinates $r, \theta$ are given in terms of the stresses $\sigma_{r r}, \sigma_{\theta \theta}$ and $\tau_{\text {rz }}$ as: $[9,10]$

$$
\begin{array}{r}
\sigma_{r}=\frac{1}{r^{2}} \frac{\partial^{2} \phi}{\partial \theta^{2}}+\frac{1}{r} \frac{\partial \phi}{\partial r} \\
\sigma_{\theta}=\frac{\partial^{2} \phi}{\partial r^{2}} \\
\tau_{r \theta}=-\frac{1}{r} \frac{\partial^{2} \phi}{\partial r \partial \theta}+\frac{1}{r^{2}} \frac{\partial \phi}{\partial \theta} \\
\tau_{r \theta}=-\frac{\partial}{\partial r}\left(\frac{1}{r} \frac{\partial \phi}{\partial \theta}\right)
\end{array}
$$

where $\phi(r, \theta)$ is the Airy's stress function in plane polar coordinates, $r, \theta$ are the radial and transverse coordinates of the plane polar coordinate system, $\sigma_{r r}, \sigma_{\theta \theta}$, are the normal stresses in the plane polar coordinate system, $\tau_{r \theta}$, is the shear stress in the plane polar coordinate system.

The Laplace and the biharmonic operators are respectively given as:

$$
\begin{gathered}
\nabla^{2}=\frac{\partial^{2}}{\partial r^{2}}+\frac{1}{r} \frac{\partial}{\partial r}+\frac{1}{r^{2}} \frac{\partial^{2}}{\partial \theta^{2}} \\
\nabla^{4}=\nabla^{2} \nabla^{2}=\left(\frac{\partial^{2}}{\partial r^{2}}+\frac{1}{r} \frac{\partial}{\partial r}+\frac{1}{r^{2}} \frac{\partial^{2}}{\partial \theta^{2}}\right)\left(\frac{\partial^{2}}{\partial r^{2}}+\frac{1}{r} \frac{\partial}{\partial r}\right. \\
\left.+\frac{1}{r^{2}} \frac{\partial^{2}}{\partial \theta^{2}}\right)
\end{gathered}
$$

The field equations of elasticity theory are solved subject to the traction boundary conditions.

\subsection{Advantages of the Displacement Potential Function Method}

The Navier equations represent the displacement formulation of the field governing equations of three dimensional elasticity problems; and can be expressed for axisymmetric problems. The equations are unwieldly and complicated because they are three coupled partial differential equations in terms of the three unknown displacement components. The displacement formulation can be simplified by representing the displacement field using harmonic potential functions. This decouples the originally coupled Navier differential equations. There are many harmonic potential functions that can be used. The Papkovich - Neuber potential functions are harmonic functions that can be used to decouple the Navier differential equations. 
3. THEORETICAL FRAMEWORK AND METHODOLOGY

\subsection{Governing Equations of Axisymmetric Elasticity Problems}

Axisymmetric elasticity problems are problems involving finding stresses and strains instructures, due to loads that exhibit rotational symmetry about the circumferential coordinate $\theta$.If the two conditions of axially symmetric geometry and axisymmetric loading are satisfied, the response of the structure will be axisymmetric (or radially symmetric).Then,the response/behaviour of the structure- displacement, strains and stresses - are independent of the circumferential coordinate.

The governing equationsare presented using the three dimensional cylindrical coordinate system $(r, z, \theta)$ where $r$ is the radial coordinate which is the distance from the axis of symmetry, $r \geq 0$; $z$ is the axial coordinate, directed along the axis of symmetry, and $\theta$ is the circumferential coordinate. The displacement field $u(r, z)$ is a function of the $r$ and $z$ coordinates, defined by the two components:

$$
u(r, z)=\left(u_{r}(r, z), u_{z}(r, z), 0\right)
$$

where $u_{r}(r, z)$ is the radial displacement, $\mathrm{u}_{z}(\mathrm{r}, \mathrm{z})$ is the axial displacement, and $u_{\theta}$ the circumferential displacement is zero due to rotational symmetry.

The strain tensor in cylindrical coordinates is represented by the symmetric matrix:

$$
\varepsilon=\left(\begin{array}{lll}
\varepsilon_{r r} & \varepsilon_{r z} & \varepsilon_{r \theta} \\
\varepsilon_{r z} & \varepsilon_{z z} & \varepsilon_{z \theta} \\
\varepsilon_{r \theta} & \varepsilon_{z \theta} & \varepsilon_{\theta \theta}
\end{array}\right)
$$

Due to the axisymmetric deformations, $\varepsilon_{r \theta}$ and $\varepsilon_{z \theta}$ vanish, and the strain tensor has only four distinct (non zero) components, thus:

Where

$$
\begin{gathered}
\varepsilon=\left(\begin{array}{ccc}
\varepsilon_{r r} & \varepsilon_{r z} & 0 \\
\varepsilon_{r z} & \varepsilon_{z z} & 0 \\
0 & 0 & \varepsilon_{\theta \theta}
\end{array}\right) \\
\operatorname{or}\left(\begin{array}{c}
\varepsilon_{r r} \\
\varepsilon_{z z} \\
\varepsilon_{\theta \theta}
\end{array}\right)
\end{gathered}
$$

$$
\gamma_{r z}=\varepsilon_{r z}+\varepsilon_{z r}=2 \varepsilon_{r z}(38)
$$

The stress tensor in cylindrical coordinates is given by the symmetric matrix:

$$
\sigma=\left(\begin{array}{lll}
\sigma_{r r} & \sigma_{r z} & \sigma_{r \theta} \\
\sigma_{r z} & \sigma_{z z} & \sigma_{z \theta} \\
\sigma_{r \theta} & \sigma_{z \theta} & \sigma_{\theta \theta}
\end{array}\right)
$$

Due to rotational symmetry the stress components $\sigma_{\mathrm{r} \theta}$ and $\sigma_{z \theta}$ vanish, and the stress tensor for axially symmetric elasticity problems simplify to:

$$
\begin{array}{r}
\sigma=\left(\begin{array}{ccc}
\sigma_{r r} & \sigma_{r z} & o \\
\sigma_{r z} & \sigma_{z z} & o \\
o & o & \sigma_{\theta \theta}
\end{array}\right) \\
\text { Or, } \sigma=\left(\begin{array}{c}
\sigma_{r r} \\
\sigma_{z z} \\
\sigma_{\theta \theta} \\
\sigma_{r z}
\end{array}\right)
\end{array}
$$

The governing partial differential equation for axisymmetric elasticity problems are the strain- displacement equations, the differential equations of equilibrium and the material constitutive laws, subject to the displacement and stress boundary conditions.

\subsubsection{Kinematic equations}

For small-displacement, linear elastic behaviour, the strain-displacement equations for axisymmetric elasticity problems are $[11,12]$.

$$
\begin{aligned}
\varepsilon_{r r} & =\frac{\partial u_{r}}{\partial r} \\
\varepsilon_{z z} & =\frac{\partial u_{z}}{\partial z} \\
\varepsilon_{\theta \theta} & =\frac{u_{r}}{r} \\
Y_{r z}=\frac{\partial u_{r}}{\partial z}+\frac{\partial u_{z}}{\partial r}=\varepsilon_{r z}+\varepsilon_{z r} & =2 \varepsilon_{r z}
\end{aligned}
$$

In matrix form,

$$
\varepsilon=\left(\begin{array}{c}
\varepsilon_{r r} \\
\varepsilon_{\mathrm{zz}} \\
\varepsilon_{\theta \theta} \\
\gamma_{r z}
\end{array}\right)=\left(\begin{array}{c}
\varepsilon_{r r} \\
\varepsilon_{\mathrm{zz}} \\
\varepsilon_{\theta \theta} \\
2 \varepsilon_{r z}
\end{array}\right)=\left(\begin{array}{cc}
\partial / \partial r & 0 \\
0 & \partial / \partial z \\
1 / r & 0 \\
\partial / \partial \mathrm{z} & \partial / \partial r
\end{array}\right)\left(\begin{array}{l}
u_{r} \\
u_{\mathrm{z}}
\end{array}\right)
$$

\subsubsection{Material constitutive law}

For linear hyperelastic materials, and neglecting thermal and prestress effects, the most general constitutive(stress-strain)equation for axisymmetric elasticity has the general form:[11][12]

$$
\sigma=\left(\begin{array}{c}
\sigma_{r r} \\
\sigma_{z z} \\
\sigma_{\theta \theta} \\
\sigma_{r z}
\end{array}\right)=\left(\begin{array}{ccc}
E_{11} E_{12} E_{13} E_{14} \\
E_{12} E_{22} E_{23} E_{24} \\
E_{13} E_{23} E_{33} & o \\
E_{14} E_{24} & o & E_{44}
\end{array}\right)\left(\begin{array}{c}
\varepsilon_{r r} \\
\varepsilon_{z z} \\
\varepsilon_{\theta \theta} \\
r_{r z}
\end{array}\right)=E \varepsilon
$$

where $E_{11}, \ldots E_{44}$ are the coefficients of the stress-stain matrix, andwhere it is observed that the cross-coupling between the shear strain and the hoop stress must vanish in axisymmetry, leading to $\mathrm{E}_{34}=\mathrm{E}_{43}=0$.

For isotropic linear elastic materialswith Young's Modulus, $\mathrm{E}$ and Poisson's ratio $\mu$, the stress-strain law simplifies for axisymmetrical problems to:[11][13]

$$
\sigma=\hat{E} \varepsilon
$$

where, $\hat{E}$ is a matrixgiven by:

$$
\hat{E}=\frac{E}{(1+\mu)(1-2 \mu)}\left[\begin{array}{cccc}
(1-\mu) & \mu & \mu & 0 \\
\mu & (1-\mu) & \mu & 0 \\
\mu & \mu & (1-\mu) & 0 \\
0 & 0 & 0 & \frac{1}{2}(1-2 \mu)
\end{array}\right]
$$

\subsubsection{Differential Equations of Equilibrium}

The general three dimensional differential equations of staticequilibrium in cylindrical coordinates are the system of three equations:[12] [13]

$$
\begin{gathered}
\frac{1}{r} \frac{\partial}{\partial r}\left(r \sigma_{r r}\right)+\frac{\partial}{r \partial \theta} \sigma_{r \theta}+\frac{\partial}{\partial z} \sigma_{r z}-\frac{\sigma_{\theta \theta}}{r}+F_{r}=0 \\
\frac{1}{r} \frac{\partial}{\partial r}\left(r \sigma_{z r}\right)+\frac{1}{r} \sigma_{z \theta}+\frac{\partial \sigma_{z z}}{\partial z}+F_{z}=0 \\
\frac{1}{r^{2}} \frac{\partial}{\partial r}\left(r^{2} \sigma_{\theta r}\right)+\frac{1}{r} \frac{\partial}{\partial \theta} \sigma_{\theta \theta}+\frac{\partial}{\partial z} \sigma_{\theta z}+F_{\theta}=0
\end{gathered}
$$


Where $\mathrm{F}_{\mathrm{r}}, \mathrm{F}_{\mathrm{z}} \mathrm{andF}_{\theta}$ are the components of the body force field in the radial, axial and circumferential coordinate directions, respectively.

For axisymmetric cases, the differential equations of staticequilibrium simplify to:

$$
\begin{aligned}
& \frac{1}{r} \frac{\partial}{\partial r}\left(r \sigma_{r r}\right)+\frac{\partial}{\partial z} \sigma_{r z}-\frac{\sigma_{\theta \theta}}{r}+F_{r}=0 \\
& \frac{1}{r} \frac{\partial}{\partial r}\left(r \sigma_{z r}\right)+\frac{\partial}{\partial z} \sigma_{z z}+F_{z}=0
\end{aligned}
$$

The third equation of equilibrium is identically satisfied if $\mathrm{F}_{\theta}=0$, because $\sigma_{\theta \mathrm{r}}=\sigma_{\theta \mathrm{z}}=0$, and $\sigma_{\theta \theta}$ is independent of $\theta$. If $\mathrm{F}_{\theta} \neq 0$, the problem of elasticity cannot be axially symmetric.

\subsection{Displacement Potential Function Formulation of Axisymmetric Elasticity Problems}

For axially symmetric elasticity problems, the displacement field $u$ can be represented as Equation (34), where, $\mathrm{u}_{\mathrm{r}}(\mathrm{r}, z)$ is the radial component of the displacement, $u_{z}(r, z)$ is the $z$ component of the displacement, and $u(r, z)$ does not depend on the $\theta$ component. Thus:

$$
u_{\theta}(r, z)=0
$$

Axially symmetric elasticity problems can be defined using displacement potential functions. The Boussinesq Papkovich - Neuber displacement potential functions $\varphi(\mathrm{r}, \mathrm{z})$ and $\Psi(\mathrm{r}, \mathrm{z})$ are defined in terms of the displacementsu $\mathrm{r}_{\mathrm{r}}(\mathrm{r}, \mathrm{z})$ and $\mathrm{u}_{\mathrm{z}}(\mathrm{r}, \mathrm{z})$ as follows:

$$
\begin{array}{r}
\mathrm{u}_{r}(\mathrm{r}, \mathrm{z})=\frac{-1}{4(1-\mu)} \frac{\partial \phi(r, \mathrm{z})}{\partial r} \\
\mathrm{u}_{\mathrm{z}}(\mathrm{r}, \mathrm{z})=\Psi(\mathrm{r}, \mathrm{z})-\frac{1}{4(1-\mu)} \frac{\partial \phi(r, \mathrm{z})}{\partial \mathrm{z}}
\end{array}
$$

$$
\text { Where, } \quad \phi(r, z)=v(r, z)+z \Psi(r, z)
$$

and, $\mu$ is the Poisson's ratio.

The Boussinesq - Papkovich - Neuber displacement functions $\varphi(\mathrm{r}, \mathrm{z})$ and $\Psi(\mathrm{r}, \mathrm{z})$ are harmonic functions, and thus satisfy the Laplace's equationsin the cylindrical coordinate system.Thus

$$
\begin{aligned}
\nabla^{2} \varphi(r, z) & =0 \\
\nabla^{2} \Psi(r, z) & =0
\end{aligned}
$$

Where $\nabla^{2}$, the Laplacian operator in cylindrical coordinate system,is given by:

$$
\nabla^{2}=\frac{\partial^{2}}{\partial r^{2}}+\frac{1 \partial}{r \partial r}+\frac{\partial^{2}}{\partial z^{2}}
$$

The normal strains, $\varepsilon_{r r}, \varepsilon_{\theta \theta}, \varepsilon_{z z}$ are expressed in terms of the displacementpotential functions using the strain displacement relations for small displacement assumptions.

Thus,

$$
\begin{gathered}
\varepsilon_{r r}=\frac{\partial u}{\partial r}=\frac{-1}{4(1-\mu)} \frac{\partial^{2} \phi}{\partial r^{2}} \\
\varepsilon_{\theta \theta}=\frac{u_{r}}{r}=\frac{-1}{4(1-\mu)} \frac{1}{r} \frac{\partial \phi}{\partial r}
\end{gathered}
$$

$$
\begin{gathered}
\varepsilon_{z z}=\frac{\partial u_{z}}{\partial z}=\frac{\partial \Psi}{\partial z}-\frac{1}{4(1-\mu)} \frac{\partial^{2} \phi}{\partial z^{2}} \\
\gamma_{r z}=\frac{\partial u_{r}}{\partial z}+\frac{\partial u_{z}}{\partial r} \\
\gamma_{r z}=\frac{-2}{4(1-\mu)} \frac{\partial^{2} \phi}{\partial r \partial z}+\frac{\partial \Psi}{\partial r} \\
\varepsilon_{r z}=\frac{1}{2} \gamma_{r z}=\frac{-1}{4(1-\mu)}\left(\frac{\partial^{2} \phi}{\partial r \partial z}-2(1-\mu) \frac{\partial \Psi}{\partial r}\right) \\
\varepsilon_{r \theta}=\frac{1}{2}\left(\frac{\partial u_{r}}{\partial \theta}+\frac{\partial u_{\theta}}{\partial r}\right)=0 \\
\varepsilon_{z \theta}=\frac{1}{2}\left(\frac{\partial u_{z}}{\partial \theta}+\frac{\partial u_{\theta}}{\partial r}\right)=0
\end{gathered}
$$

The volumetric strain, $\varepsilon_{\mathrm{v}}$ is:

$$
\begin{array}{r}
\varepsilon_{v}=\varepsilon_{r r}+\varepsilon_{\theta \theta}+\varepsilon_{z z} \\
\varepsilon_{v}=\frac{-1}{4(1-\mu)}\left(\frac{\partial^{2} \phi}{\partial r^{2}}+\frac{1}{r} \frac{\partial \phi}{\partial r}+\frac{\partial^{2} \phi}{\partial z^{2}}\right)+\frac{\partial \Psi}{\partial z} \\
\varepsilon_{v}=\frac{-1}{4(1-\mu)}\left(\nabla^{2} \phi\right)+\frac{\partial \Psi}{\partial z} \\
\varepsilon_{v}=\frac{-1}{4(1-\mu)}\left(\nabla^{2}(\varphi+z \Psi)\right)+\frac{\partial \Psi}{\partial z} \\
\varepsilon_{v}=\frac{1-2 \mu}{2(1-\mu)} \frac{\partial \Psi}{\partial z}
\end{array}
$$

from Equations (59) and (60).The normal stresses $\sigma_{r r}, \sigma_{\theta \theta}, \sigma_{z z}$ are found from the stress - strain laws.Thus,

$$
\sigma_{r r}=\lambda \varepsilon_{v}+2 G \varepsilon_{r r}=\lambda \frac{\partial \Psi}{\partial z}-\frac{2 G}{4(1-\mu)} \frac{\partial^{2} \phi}{\partial r^{2}}
$$

Where $\lambda$ which is the Lame constant:

$$
\lambda=\frac{2 \mu G}{1-2 \mu}
$$

Where

$$
\begin{gathered}
\sigma_{r r}=\frac{-G}{2(1-\mu)}\left(\frac{\partial^{2} \phi}{\partial r^{2}-}{ }^{2 \mu \partial \Psi} \frac{\partial \Psi}{\partial z}\right) \\
\sigma_{\theta \theta}=\lambda \varepsilon_{V}+2 G \varepsilon_{\theta \theta} \\
\sigma_{\theta \theta}=\frac{-G}{2(1-\mu)}\left(\frac{1 \partial \phi}{r \partial r}-\frac{2 \mu \partial \Psi}{\partial z}\right) \\
\sigma_{\mathrm{zz}}=\lambda \varepsilon_{V}+2 G \varepsilon_{\mathrm{zz}} \\
\sigma_{\mathrm{zz}}=\frac{-G}{2(1-\mu)}\left(\frac{\partial^{2} \phi}{\partial z^{2}}-2(2-\mu) \frac{\partial \Psi}{\partial z}\right) \\
\sigma_{r z}=\frac{-G}{2(1-\mu)}\left(\frac{\partial^{2} \phi}{\partial r \partial z}-2(1-\mu) \frac{\partial \Psi}{\partial r}\right) \\
\sigma_{r \theta}=0 \\
\sigma_{z \theta}=0
\end{gathered}
$$

\section{RESULTS}

\subsection{Application of Bessel Functions}

For the problem of point load $\mathrm{P}$ acting at the origin $(0,0,0)$ of a semi-infinite soil presented in Figure 1 , the boundary conditions are given from the theory of Dirac delta function representation in cylindrical coordinates system of point load, $P$ and the relationship between the Dirac delta function $\delta(r)$ and the Bessel function as: 


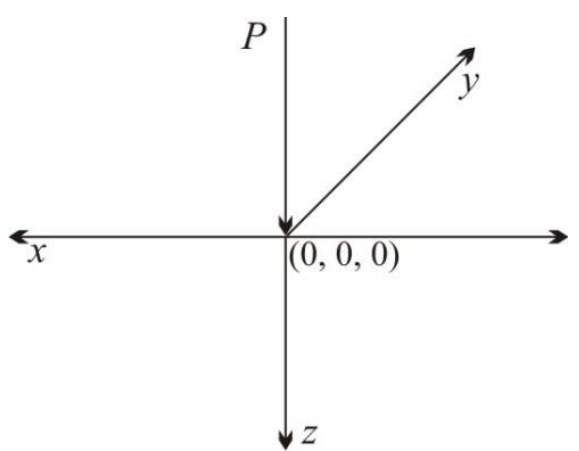

Figure 1: Point load Pacting at the origin of a semi-infinite (half space) soil.

$$
\begin{gathered}
\sigma_{\mathrm{zz}}(r, o)=-\frac{P \delta(r)}{2 \pi r}=\frac{-P}{2 \pi} \int_{o}^{\infty} k J_{o}(k r) d k \\
\text { And, } \sigma_{r z}(r, o)=0
\end{gathered}
$$

Where,

$$
\mathrm{J}_{o}=\mathrm{J}_{o}(k r)
$$

$J_{0}(k r)$ is the Bessel function of order zero, and of the first kind that is the solution of the differential equation

$$
\left(\nabla_{r}^{2}+k^{2}\right) J_{o}(k r)=0
$$

$$
\text { Where, } \nabla_{r}^{2}=\frac{\partial^{2}}{\partial r^{2}}+\frac{1}{r} \frac{\partial}{\partial r}
$$

TheBoussinesq- Papkovich - $\quad$ Neuber displacementpotential functions $\phi$ and $\Psi$ that satisfy the boundary conditions can be expressedusing Bessel functionsas:

$$
\begin{array}{r}
\phi(r, z)=\int_{0}^{\infty}\left(c_{1}+k z c_{2}\right) e^{-k \mathrm{z}} J_{o}(k r) d k \\
\Psi(r, \mathrm{z})=\int_{0}^{\infty} c_{3} e^{-k \mathrm{z}} J_{o}(k r) d k
\end{array}
$$

Where, $c_{1}(k), c_{2}(k)$ and $c_{3}(k)$ are all unknown functions of the parameter $k$. These three unknown functions will be determined from the boundary conditions and from Equations (60) and (92).

$$
\begin{gathered}
\nabla^{2} \phi=2 \frac{\partial \Psi}{\partial z} \\
\nabla^{2} \int_{0}^{\infty} c_{3} e^{-k z} J_{o}(k r) d k=0 \\
\int_{0}^{\infty} c_{3} \nabla^{2}\left(e^{-k z} J_{o}(k r)\right) d k=0 \\
=c_{3} \int_{0}^{\infty} \nabla^{2}\left(e^{-k z} J_{0}(k r)\right) d k \\
\nabla^{2}\left(e^{-k z} J_{o}(k r)\right)=0 \\
\operatorname{From}^{2} \nabla^{2} \nabla^{2} \phi=0 \\
\nabla^{2} \nabla^{2} \int_{0}^{\infty}\left(c_{1}+k z c_{2}\right) e^{-k z} J_{o}(k r) d k=0 \\
\int_{0}^{\infty}\left(c_{1}+k z c_{2}\right) e^{-k z} J_{o}(k r) d k \\
=2 \frac{\partial}{\partial z} \int_{0}^{\infty} c_{3} e^{-k z} J_{o}(k r) d k
\end{gathered}
$$

$$
\begin{gathered}
\int_{0}^{\infty}-2 k^{2} c_{2} e^{-k z} J_{o}(k r) d k \\
=2 \int_{0}^{\infty}-k c_{3} e^{-k z} J_{o}(k r) d k \\
\sigma_{r z}=\frac{-G}{2(1-\mu)}\left\{\frac{\partial^{2}}{\partial r \partial z} \int_{0}^{\infty}\left(c_{1}+k z c_{2}\right) e^{-k z} J_{o}(k r) d k\right. \\
-2(1 \\
\left.\frac{-G}{2(1-\mu)} \int_{0}^{\infty}\left(-c_{1} k e^{-k z}+k c_{2}(-k z+1) e^{-k z}\right)-k J_{1}(k r)\right) d k \\
-2(1-\mu) \int_{0}^{\infty}\left(k c_{2} e^{-k z}\right)-k J_{1}(k r) d k=\sigma_{r z} \quad(102)
\end{gathered}
$$

For $z=0$,

$$
c_{1}=-(1-2 \mu) c_{2}
$$

Then the Boussinesq- Papkovich - Neuber displacementpotential functions can be expressed in terms of one unknown constant, $\mathrm{c}_{2}$, as follows:

$$
\begin{gathered}
\phi(r, \theta)=\int_{0}^{\infty} c_{2}(k z-(1-2 \mu)) e^{-k z} J_{o}(k r) d k \\
\Psi(r, \theta)=\int_{0}^{\infty} k c_{2} e^{-k z} J_{o}(k r) d k \\
\sigma_{\mathrm{zz}}=\frac{-G}{2(1-\mu)}\left\{\frac { \partial ^ { 2 } } { \partial z ^ { 2 } } \int _ { 0 } ^ { \infty } \left(c_{2}(k z-(1-2 \mu)) e^{-k z} J_{o}(k r) d k\right.\right. \\
\left.-2(2-\mu) \frac{\partial}{\partial z} \int_{0}^{\infty} k c_{2} e^{-k z} J_{o}(k r) d k\right\}
\end{gathered}
$$

$$
\begin{aligned}
\frac{-G}{2(1-\mu)}\left\{\int_{0}^{\infty} c_{2}(\right. & \left.k z-(1-2 \mu) k^{2} e^{-k z}-2 k^{2} e^{-k z}\right) \\
\times & J_{o}(k r) d k \\
& \left.-2(2-\mu) \int_{0}^{\infty}-k^{2} c_{2} e^{-k z} J_{o}(k r) d k\right\}\left.\right|_{r, z=0} \\
& =\frac{-P}{2 \pi} \int_{0}^{\infty} k J_{o}(k r) d k
\end{aligned}
$$

At $z=0$, for any $r$,

$$
\begin{gathered}
c_{2}=\frac{P \cdot(1-\mu)}{k G \pi} \\
c_{3}=k c_{2}=\frac{P \cdot(1-\mu)}{G \pi} \\
c_{1}=-(1-2 \mu) c_{2}=\frac{-(1-2 \mu)(1-\mu) P}{k G \pi}
\end{gathered}
$$

Thus

$$
\Psi=\frac{P(1-\mu)}{G \pi} \int_{0}^{\infty} e^{-k z} J_{o}(k r) d k
$$




$$
\begin{gathered}
\phi=\frac{P(1-\mu)}{k G \pi} \int_{0}^{\infty}(k z-(1-2 \mu)) e^{-k z} J_{o}(k r) d k \\
\frac{\partial \phi}{\partial r}=\frac{P(1-\mu)}{G \pi} \int_{0}^{\infty}((1-2 \mu)-k z) e^{-k z} J_{1}(k r) d k \\
\frac{\partial \phi}{\partial z}=\frac{P(1-\mu)}{k G \pi} \int_{0}^{\infty}(2(1-2 \mu) \\
\frac{\partial \phi}{\partial z}=\frac{P(1-\mu)}{G \pi} \int_{0}^{\infty}(2(1-2 \mu) \\
-k z) e^{-k z} J_{o}(k r) d k
\end{gathered}
$$

The integrals involving $e^{-k z} J_{n}(k r)$ as integrand can be evaluated using the formula:

$$
\int_{0}^{\infty} e^{-k \mathrm{z}} J_{n}(k r) d k=\frac{r^{-n}(R-\mathrm{z})^{n}}{R} ; \quad \mathrm{n}=0,1,2,3
$$

Where, $\mathrm{R}^{2}=\mathrm{r}^{2}+\mathrm{z}^{2}, \mathrm{z} \geq 0$

$J_{n}(k r), n \geq 0$ is the Bessel function of the first kind (of order zero), which is the solution to the differential equation:

$$
\left[\nabla_{r}^{2}+\left(k^{2}-\frac{n^{2}}{r^{2}}\right)\right] J_{n}(k r)=0
$$

Thus, the integrals occurring in the expressions forthe Boussinesq - Papkovich - Neuber displacement potential functions $\phi$ and $\Psi$ and their derivatives can be expressed in terms of common mathematical functions.

$$
\begin{array}{r}
\int_{0}^{\infty} e^{-k z} J_{o}(k r) d k=\frac{r^{-0}(R-z)^{0}}{R}=\frac{1}{R}(118) \\
\int_{0}^{\infty} e^{-k z} J_{1}(k r) d k=\frac{r^{-1}(R-z)^{1}}{R}=\frac{r}{R(R+z)}(119) \\
\frac{\partial \phi}{\partial r}=\frac{P(1-\mu)}{G \pi} \frac{1}{R}(120) \\
\frac{P(1-\mu)}{\partial \pi}\left(\frac{\partial \phi}{R(R+z)}+\frac{\partial(1-\mu)}{\pi G}\left(\frac{2(1-\mu)}{R}+\frac{z \partial}{\partial z} \frac{1}{R}\right)(122)\right. \\
\frac{\partial(R+z)}{R(R+z)}-\frac{1}{R^{3}}(123) \\
\frac{\partial \phi}{\partial r}=\frac{P(1-\mu)}{G \pi}\left(\frac{(1-2 \mu) r}{R(R+z)}-\frac{r z}{R^{3}}\right) \\
\frac{\partial \phi}{\partial z}=\frac{P(1-\mu)}{G \pi}\left(\frac{2(1-\mu)}{R}-\frac{z^{2}}{R^{3}}\right)
\end{array}
$$

Hence,

$$
\begin{aligned}
& u_{r}=\frac{P}{4 G \pi} \frac{r}{R}\left(\frac{z}{R^{2}}-\frac{(1-2 \mu)}{R+z}\right) \\
& u_{z}=\frac{P}{4 G \pi} \frac{1}{R}\left(\frac{z^{2}}{R^{2}}+2(1-\mu)\right)
\end{aligned}
$$

By differentiation,

$$
\begin{gathered}
\frac{\partial^{2} \phi}{\partial r^{2}}=\frac{P(1-\mu)}{G \pi}\left[\frac{z}{R^{3}}\left((3-2 \mu)-\frac{3 z^{2}}{R^{2}}\right)\right. \\
\left.-(1-2 \mu) \frac{1}{R(R+z)}\right] \\
\frac{\partial^{2} \phi}{\partial z^{2}}=\frac{P(1-\mu)}{G \pi}\left(\frac{z}{R^{3}}\left[\frac{3 z^{2}}{R^{2}}-2(2-\mu)\right]\right) \\
\frac{\partial \Psi}{\partial z}=\frac{-P(1-\mu)}{G \pi} \frac{r}{R^{3}}
\end{gathered}
$$

Then by substitution, the normal strain components $\varepsilon_{r r}$, $\varepsilon_{\theta \theta}$ and $\varepsilon_{z z}$ and the shear strain components $\varepsilon_{\mathrm{r} \theta}$ become:

$$
\begin{gathered}
\varepsilon_{r r}=-\frac{P}{4 \pi G}\left(\frac{z}{R^{3}}\left((3-2 \mu)-\frac{3 z^{2}}{R^{2}}\right)\right. \\
\left.-(1-2 \mu) \frac{1}{R(R+z)}\right) \\
\varepsilon_{\theta \theta}=-\frac{P}{4 \pi G}\left\{(1-2 \mu) \frac{1}{R(R+z)}-\frac{z}{R^{3}}\right\} \\
\varepsilon_{z z}=-\frac{P}{4 \pi G}\left\{\frac{z}{R^{3}}\left(\frac{3 z^{2}}{R^{2}}-2 \mu\right)\right\} \\
\varepsilon_{r z}=-\frac{3 P}{4 \pi G} \frac{r z^{2}}{R^{5}}
\end{gathered}
$$

The normal stress components $\sigma_{r r}, \sigma_{\theta \theta}$ and $\sigma_{z z}$ are:

$$
\begin{gathered}
\sigma_{r r}=\frac{P}{2 \pi R^{2}}\left(-\frac{3 r^{2} z}{R^{3}}+\frac{(1-2 \mu) R}{R+z}\right) \\
=\frac{P}{2 \pi}\left(\frac{-3 z r^{2}}{R^{5}}+\frac{(1-2 \mu)}{R(R+z)}\right) \\
\sigma_{\theta \theta}=\frac{(1-2 \mu) P}{2 \pi R^{2}}\left(\frac{z}{R}-\frac{R}{R+z}\right) \\
=\frac{(1-2 \mu) P}{2 \pi}\left(\frac{z}{R^{3}}-\frac{1}{R(R+z)}\right) \\
\sigma_{\mathrm{zz}}=-\frac{3 P}{2 \pi} \frac{z^{3}}{R^{5}} \\
\sigma_{r z}=\frac{3 P}{2 \pi} \frac{z^{2} r}{R^{5}}
\end{gathered}
$$

The solution for the displacement fields and stress fields can be obtainedin terms of the threedimensional Cartesian coordinate system using the transformations:

$$
\begin{gathered}
u_{x}=u_{r} \cos \theta \\
v=u_{y}=u_{r} \sin \theta \\
w=u_{z} \\
\sigma_{x z}=\sigma_{r z} \cos \theta \\
\sigma_{y z}=\sigma_{r z} \sin \theta \\
\sigma_{z z}=\sigma_{z z} \\
\sigma_{x x}=\sigma_{r r} \cos ^{2} \theta+\sigma_{\theta \theta} \sin ^{2} \theta \\
\sigma_{y y}=\sigma_{r r} \sin ^{2} \theta+\sigma_{\theta \theta} \cos ^{2} \theta \\
\sigma_{x y}=1 / 2\left(\sigma_{r r}-\sigma_{\theta \theta}\right) \sin 2 \theta \\
\text { Where, } \sin \theta=\mathrm{y} / \mathrm{R} \\
\cos \theta=x / R
\end{gathered}
$$

Then, 


$$
\begin{aligned}
& u_{x}=\frac{P}{4 \pi G} \frac{x}{R}\left\{\frac{z}{R^{2}}-\frac{(1-2 \mu)}{R+z}\right\} \\
& =\frac{P(1+\mu)}{2 \pi E}\left(\frac{x z}{R^{3}}-\frac{(1-2 \mu) x}{R(R+z)}\right) \\
& v=u_{y}=\frac{P}{4 \pi G} \frac{y}{R}\left\{\frac{z}{R^{2}}-\frac{(1-2 \mu)}{R+z}\right\} \\
& =\frac{P(1+\mu)}{2 \pi E}\left(\frac{y z}{R^{3}}-\frac{(1-2 \mu) y}{R(R+z)}\right) \\
& u_{z}=w=\frac{P}{4 \pi G R}\left\{\frac{z^{2}}{R^{2}}+2(1-\mu)\right\} \\
& =\frac{P(1+\mu)}{2 \pi E}\left(\frac{z^{2}}{R^{3}}+\frac{2(1-\mu)}{R}\right) \\
& \sigma_{x x}=\frac{-P}{2 \pi R^{2}}\left\{\frac{3 x^{2} z}{R^{3}}\right. \\
& -(1-2 \mu)\left(\frac{z}{R}-\frac{R}{R+z}\right. \\
& \left.\left.+\frac{x^{2}(2 R+z)}{R(R+z)^{2}}\right)\right\} \\
& \sigma_{y y}=\frac{-P}{2 \pi R^{2}}\left\{\frac{3 y^{2} z}{R^{3}}\right. \\
& -(1-2 \mu)\left(\frac{x}{R}-\frac{R}{R+z}\right. \\
& \left.\left.+\frac{y^{2}(2 R+z)}{R(R+z)^{2}}\right)\right\} \\
& \sigma_{\mathrm{zz}}=\frac{-P}{2 \pi R^{2}} \frac{3 \mathrm{z}^{3}}{R^{3}}=\frac{-3 P z^{3}}{2 \pi R^{5}} \\
& \sigma_{x y}=\frac{-P}{2 \pi R^{2}} x y\left(\frac{3 z}{R^{3}}-\frac{(1-2 \mu)(2 R+z)}{R(R+z)^{2}}\right)
\end{aligned}
$$

\section{DISCUSSION}

This work has successfully presented the governing partial differential equations for axisymmetric elasticity problems of linearly elastic, homogeneous, isotropic soils; represented the governing field equations using the Boussinesq - Papkovich - Neuberpotential function, and then solved the problem of homogenous soil of semiinfinite extent loaded by a point load $P$ applied at the origin using Boussinesq - Papkovich - Neuber functions assumed in the form of Bessel functions of order zero, and of the first kind.

The field equations of axisymmetric elasticity for homogeneous, isotropic materials are given as Equations (42) (45), (48) and (53- 54). The Boussinesq Papkovich - Neuber potential function formulation of the field equations are presented as Equations (62)- (69) and (75) - (84). The Boussinesq - Papkovich - Neuber potential functions were expressed using Bessel functions in the form of Equations(90) and (91) such that they contained unknown parameters $\mathrm{c}_{1}(\mathrm{k}), \mathrm{c}_{2}(\mathrm{k})$ and $c_{3}(k)$ which were determined using the boundary conditions. The unknown parameters were determined as Equations (108), (109) and (110), yielding complete solutionsfor the potential functions.The displacement fields were determined as Equations (127) and (128) using cylindrical coordinates.The stress components were found as equations (136) - (139), using cylindrical coordinates.

The displacement fields were determined using Cartesian coordinates as Equations (151)-(153) and the stress fields expressed in terms of Cartesian coordinates as Equations(154) - (157).

The expressions found for the stress components and the displacement field were exactly identical with Bousinesq's solutions [14] and presented in most books on soil mechanics [15].

\section{CONCLUSION}

The following conclusions are made from the study:

(i) the axisymmetric elasticity problem of a half-space considered homogeneous, isotropic and linear elastic has been successfully formulated using the Boussinesq - Papkovich - Neuber displacement potential functions.

(ii) the displacements and stresses were found by assuming the Boussinesq - Papkovich - Neuber displacement potential functions in terms of Bessel functions of order zero and of the first kind and applying the boundary conditions.

(iii) the displacements and stresses $\sigma_{r r}, \sigma_{\theta \theta}, \sigma_{z z}$ obtained for a point load acting at the origin of a semi-infinite elastic space were found to be exactly the same as those obtained by the use of stress potential function methods and presented in literature.

(iv) the effectiveness and generality of the displacement potential function method for analysis of elastic halfspace problems is thus illustrated.

\section{REFERENCES}

[1] Randolph M. and Gourvenec. Offshore Geotechnical Engineering with Contributions from White D. and Cassidy M. Spoon Press, New York, USA and Canada.

[2] Constantinescu E. and Bojden M. On computing special functions in marine engineering. Modern Technologies in Industrial Engineering (Mod Tech 2015) IOP conference series: Materials Science and Engineering, 95, 2015, pp. 1-7.

[3] SaddM.H. Elasticity Theory, Application and Numerics University of Rhode Island Elsevierhttps://personalegr.Uri.edu/taggart/cour ses/ce571/Chapter 7.pptx.Retrieved 15/02/2015.

[4] Ramesh, K. Engineering Fracture Mechanics Module No 3 Lecture No 13 Displacement and Stress Formulations. Department of Applied 
Mechanics Indian Institute of Technology, Madras Text of video. Nptelrytmrac.in/112106065/leck,13;pdf.Retrieve d 10/4/2016.

[5] Muskhelishvili N.I. (Ed.), Some Basic Problems of the Mathematical Theory of Elasticity; Fundamental Equations, Plane Theory of Elasticity, Torsion and Bending Fourth, Kluwer corrected and augmented edition Moscow. Translated from the Russain by J.R.M RadokNoordhoffInternational Publishing Leyden, 1977,The Netherlands., http://book.goggle.wn/sceince >MechanismGener al.Retrieved 15/02/2015.

[6] Timoshenko, S.P. and Goodier, J. N. Theory of Elasticity, Third Edition,McGraw Hill, New York. 1970.

[7] Sokolnikoff, I. S. Mathematical Theory of Elasticity, Second Edition, Data McGraw Hill Publishing Company Ltd, Bombay, New Delhi. 1956.

[8] Ramadas Chennamsetti, 2D Theory of Elasticity mechanica.org/files/2D\%20theory\%20of\%elastici ty.pdfRetrieved 10/02/2015.

[9] Sitharam T.G. and GovindaReju L. Applied Elasticity for Engineers, Module: Elastic solutions and Applications in Geomechanics 14.139.172.204/nptel/1/CSE/web/105108070/m odule 8/lecture 17. pdf. Retrieved 10/02/2015.

[10] Hazel A. MATH 350211 Elasticity www.maths.manchester.acuk/-ahazel/MATHS. Nov.30,2015. Retrieved 8/04/2016. 2015.

[11] Palaniappan D. A general solution of equations of equilibrium in linear elasticity, Applied Mathematical Modelling, 35 (2011). pp 5494 5499 Elservier. 2011.

[12] Axisymmetric Solids, aka Solids of Revolution, http//www.colorado.edu/engineering/CAS/cours es.d/AFEMCh02d/AFEM.Ch021 pdf. Retrieved $8 / 04 / 2016$

[13] Abeyartne, R. (2012) Continuum Mechanics Volume II of Lecture Notes on The Mechanics of Elastic Solids Cambridge, http//web.mit.edu/abeyartne/lecture_notes.html 11,May, 2012.Retrieved 8/04/2016

[14] Boussinesq M.J. Application des potentialsal'etudedetequilibreet $\mathrm{du}$ mouvements des solideselastiquesGaythier-Villars Paris. 1885.

[15] Davis, R.O and Selvadurai, A.P.S Elasticity and Geomechanics Cambridge University Press. 1996. 